The breeding animals used to produce the germ-free chicken are of a White Leghorn strain, (Orthoxenic PA I2), created at our Station.

After sanitation, the eggs are hatched in protected atmosphere. On day 18 of incubation, the eggs after sterilization of the shells, are transferred into a sterile isolator. After hatching, the animals are kept in the isolator for the rest of their life. The isolators are conceived and manufactured at the Station. They are made of rigid plastic and fitted with an automatic and programmable system of conditioning, providing total working autonomy.

We produce 160 germ-free chicken twice a month (hatching rate $80 \mathrm{p} .100$ ). Controls of setrilization effect obtained have shown that eventual contaminations are mainly due to the imperfectly autoclaved feed (it seldom occurs, and only I lot out of ro was eliminated).

\title{
SPECTINOMYCIN VERSUS SPECTINOMYCIN LINCOMYCIN COMBINATION AGAINST MYCOPLASMA GALLISEPTICUM IN GHICKENS
}

\author{
Z. ZOLLI et M. GUG* \\ ABBOTT Laboratories, \\ North Chicago (Illinois) \\ * C. E. V. A., Besançon
}

To determine whether a combination product containing spectinomycin and lincomycin was more efficacious than spectinomycin alone, experiments were conducted in vivo against an experimentally induced airsacculitis associated with Mycoplasma gallisepticum (V 248). The antibiotics were administered in the drinking water for the first three days of life of the chicks and again for a 24 hours period on the tenth day of life. On the second day of life, the desired groups of chicks were infected intrathoracically with a broth culture of $M$. gallisepticum (V 248). The experiments were terminated when the chicks reached 4 weeks of age at which time the survivors were killed, group weighed and examined for air sac lesions typically associated with $M$. gallisepticum. The various antibiotics which were administered alone or in combination were as follows:

\footnotetext{
a) spectinomycin alone at $2 \mathrm{~g} / \mathrm{gal}$ activity;

b) spectinomycin at $1.33 \mathrm{~g} / \mathrm{gal}$ and erythromycin at $0.67 \mathrm{~g} / \mathrm{gal}$ activity;

c) spectinomycin at $1.33 \mathrm{~g} / \mathrm{gal}$ and lincomycin at $0.67 \mathrm{~g} / \mathrm{gal}$ activity ;

d) tylosin alone at 2 g/gal activity ;

e) spectinomycin alone at $\mathrm{r} .33 \mathrm{~g} / \mathrm{gal}$ activity ;

f) lincomycin alone at $0.67 \mathrm{~g} / \mathrm{gal}$ activity.
}

Under the very severe experimental infection induced in this trial results indicated that the birds receiving spectinomycin alone at $2 \mathrm{~g} / \mathrm{gal}$ in the drinking water showed significantly greater weight gains and significantly less severe air sac lesions than did all other medicated groups. Under the conditions of these experiments, no synergistic activity was apparent with the spectinomycin-lincomycin combination. In fact, the combination spectinomycin-lincomycin appeared only as effective as the concentration of spectinomycin which was in the combination. 